Ambient Science, 2016: Vol. 03(Sp1); 10-14

DOI:10.21276/ambi.2016.03.sp1.ta02

ambient

SCIENCE

Vol. 3(Sp1):10-14

Year 2016

\title{
Assessment of Human Resources Performance in High-tech Aviation Industries by DEMATEL Technique and Fuzzy-analytic Network Process
}

\author{
Mohsen Sadegh Amalnick', Iman Navarchi' ${ }^{2 *}$ \\ 'Departmant of Industrial Engineering College of Engineering, \\ University of Tehran, Tehran Iran \\ ${ }^{2}$ Department Industrial Engineering, Kharazmi university, \\ Tehran, Iran \\ Study Area: Mehrabad, Iran \\ Coordinate: $35^{\circ} 41^{\prime} 21^{\prime \prime} \mathrm{N} 51^{\circ} 18^{\prime} 49^{\prime \prime} \mathrm{E}$
}

Key words: Performance evaluation, Analytic hierarchy/ network processes,

\section{Introduction:}

Nowadays when it comes to reasons of significant and advancement of developed countries, one of the most important reasons in this respect is complying with predef ined standards and patterns in all fields, especially in the occupational field. We achieve such level mainly through continuous, systematic and scientific monitoring of individual's performance on every level. It could not be overruled that the process of monitoring may vary depending on occupational, social, geographical and other logical situations (Hosein, 1999). On one side where the media persons have taken the responsibility for monitoring such cases, while in another, relevant specialists and experts having a similar interest are also involved in the same job. In order to pursue it rightly high level of honesty and spunk is essential where no bias or mal-purposes should be include (Abbas, 2001).

Despite several technologies, today's world is solely dependent on the various levels of human-oriented organizations. Each organization is dependent on human resources which are absolutely required for their strategic and vital factors for existence. Most of the experts in human resources management believe that organizations have to evaluate their staff prior to every important decision such as enhancement, wage increasing, redistributing, appointing and/or dismissing. In every level, performance, talents, potentiality and effectiveness of each staff must be assessed as per required standard which is essential for the inevitable role of creativity for the existence and prosperity of organizations and to achieve targets (Janie \& Triandis, 2002). By incorporation of performance assessment system, it would be absolutely

\section{Abstract}

This study was to evaluate the performance of human resources in high-technology industries using DEMATEL and process network analysis phase. The research methodology was based on a descriptive type of data collection. The subjects of the study comprised of all employees (8887 persons) woking in Iran Air (HOMA). Total, 365 questionnaires were collected from staffs which were analyzed to evaluate the performance of employees where the integration of ANP-DEMATEL indicators was prioritized.

possible to gain a correct and comprehensive knowledge about the individuals' capabilities and loopholes which could be helpful to solve issues of organizational work relations. Hence, necessary steps to provide relevant development plans should be taken to attain huge benefits. Nevertheless, special and complex features of organizations' decree to meet the new challenges in the field of performance assessment and its importance in success of organizations, the work domain get exceed beyond common assessment methods which could be only access by some abstract factors to get an effective model, based on some behavior and attitude support (Naseer, 2000).

In the recent years, performance assessment in any organization has been widely accepted for the desired goals and actions in the field of human resources management. Assessment evolution process reveals that it was perceived merely under managerial control and functions as per classic management discussions. However,it is considered as dynamic, continuous, qualitative and mutual (manager and staff) process as the main subsystem in human resources management. Thus, performance assessment functions now are deemed beyond rewarding, emphasis on behavior support, the relation between managers and staff and optimization of human resources in organizations. Assessment with a broader vision i.e. performance management is highly emphasized these days. Performance management is known as a new phenomenon in the field of strategic management of human resources, a part of which would be performance assessment (Mark, 1999). There are many patterns and methods to assess individuals' performance. 
The important issue in their implementation is that it must be consistent with organizational conditions and provide efficient output. From a professional viewpoint, performance assessment is a proper solution for optimization of human resources. In other words, optimization and enhancement of staff capabilities must be the goal of performanceassessment (Mark, 1999).

\section{Problem Statement:}

A basic and important asset for every organization is human resources and considering its collective features, skills and capabilities it has a significant role in development and growth of organizations. Therefore, human resources may be considered as the bedrock for intellectual capitals. Since staff performance assessment is carried out to improve capabilities, to recognize weak and strong points and to adopt proper managerial decisions they require a great deal of attention (Reza, 2003a). Performance assessment of human resources leads to organizational improvement. When continuous improvement becomes a cultural tradition in an organization, it starts experiencing a meaningful progress contributed by its staff. Performance assessment determines goal achievement level in the sake of organizational satisfaction or effectiveness. Performance assessment is to determine efficiency and output of staff through maintenance and development of vital information regarding human resources (Abbas, 2005).

Performance assessment not only provides the managers correct and valid information but also increases knowledge of staff regarding themselves for better performances. It informs them about their occupational behavior and performance as well as their weak or strong points. This information may provide a proper base to improve occupational behaviors and personality of staff (Abbas, 2005). Important decision-makings by individuals or organizations is based on performance assessment results. Staff perhaps becomes more motivated to contribute to the development process if it would be designed in the right form (Michael, 2002). In order to create an organization with efficient human resources, in this field of study, there is no alternative other than attending to education, creativity, support, innovation, and motivation. In this regard, the first step is to correctly assess the performance of staff. Then, weaknesses have to be resolved and capabilities have to be developed. In other words, optimization and training of human resources should be properly planned with performanceassessment.

In the present work we selected Iran Air Company (Homa) for our field study due to the following privileges: firstly, limited number of personnel answer the questionnaire, secondly personnel answer the evaluation questionnaire casually and nonchalantly and their answers don't reflect the reality, thirdly, after the evaluation is carried out, its results has no tangible feedback on personnel. Therefore this study seeks to evaluate the performance of workforce in air industry (case study: Iran Air Company (Homa).

Thus the present study was targeted to establish the suitable technique and proper performance assessment for human resources in high-tech industries.

\section{Applied techniques:}

Analytic hierarchy/network processes (AHP, ANP): it is a process is a mathematical method to solve multi-criteria complex required in decision-making problems. This method was developed by Saaty (1980). AHP is capable of considering both qualitative and quantitative criteria in the assessment of decision options (Jafar, 2008). In this method, it is assumed that the discussed factors are independent in a hierarchal structure. The AHP method is also known as priority vector method which includes considering the priority vector regarding the highest value of paired comparison matrix and determination of factors priorities and priority order among options (Jones \& Burly, 200o). This method has been elaborated in future sections. AHP is a measurement theory about conclusion on importance and domination of priorities from paired comparison of homogeneous members regarding a common criterion. AHP is useful in the development of decision-making models through a process that includes both qualitative and quantitative components.

\section{DEMATEL techniques:}

DEMATEL technique was first proposed by Fontela and Gabus in late 1970 to solve global issues in science, politics and economy by considering expert procedures (referred by- Nejad, 2009; Zafar, 2008). DEMATEL technique is practically used to show internal relations among criteria and find the criterion with the central role in the system that affects other criteria (Assadollah, 2008; Mansour, 2008). Further, DEMATEL technique is not only applied to know the impacts of members among each other but also to determine the complex relations and form a plan of impact relationship for members (Reza, 2003; Amir, 2006).

\section{Methodologyand Results:}

Criteria and sub-criteria were recognized and accepted in two steps. The recognition step was related to a determination of criteria, sub-criteria, and options related to the studied subject. The acceptance step consisted of confirmation and modification of the recognized criteria and sub-criteria by experts. Responders were not selected randomly but deliberately among specialists and experts of Iran Air Company. Primary criteria used for confirmation in providing questionnaires were extracted from desk studies. The important criteria \& sub-criteria are presented in Table 1 : 
Table 1: Criteria and sub-criteria

\begin{tabular}{|c|c|c|}
\hline Criteria & Sub-criteria & Symbol \\
\hline 1-Reliability & $\begin{array}{l}\text { a. Punctuality } \\
\text { b. No misuse of sick leaves } \\
\text { c. No misuse of overtime } \\
\text { d. No absence } \\
\text { e. Start working on time }\end{array}$ & $\begin{array}{l}\mathrm{S}_{11} \\
\mathrm{~S}_{12} \\
\mathrm{~S}_{13} \\
\mathrm{~S}_{14} \\
\mathrm{~S}_{15}\end{array}$ \\
\hline 2-Attitude & $\begin{array}{l}\text { a. Interest in working } \\
\text { b. Commitment to organization } \\
\text { c. Attitude towards other staff } \\
\text { d. Attitude towards other prople }\end{array}$ & $\begin{array}{l}\mathrm{S}_{21} \\
\mathrm{~S}_{22} \\
\mathrm{~S}_{23} \\
\mathrm{~S}_{24}\end{array}$ \\
\hline $\begin{array}{l}\text { 3- Working } \\
\text { quality }\end{array}$ & $\begin{array}{l}\text { a. Work accomplishment } \\
\text { b. Effectiveness } \\
\text { c. Completion of work in the allotted time } \\
\text { d. Accuracy and correctness } \\
\text { e. Tidiness }\end{array}$ & $\begin{array}{r}S_{31} \\
S_{32} \\
\text { ne } S_{33} \\
S_{34} \\
S_{35}\end{array}$ \\
\hline 4- Innovation & $\begin{array}{l}\text { a. Knowing about what has to be done } \\
\text { b. Start duties without special trainings } \\
\text { c. Following working duties }\end{array}$ & $\begin{array}{l}\mathrm{S}_{41} \\
\mathrm{~S}_{42} \\
\mathrm{~S}_{43}\end{array}$ \\
\hline 5- Judgment & $\begin{array}{l}\text { a. Use of common reason } \\
\text { b. Proper decision-making at harsh times }\end{array}$ & $\begin{array}{r}\mathrm{S}_{51} \\
\mathrm{~S}_{52}\end{array}$ \\
\hline $\begin{array}{l}\text { 6-Cooperation } \\
\text { time }\end{array}$ & $\begin{array}{l}\text { S61 a. Working in demanded place and } \\
\text { b. Working in a section or department } \\
\text { c. Working with other sections or } \\
\text { departments } \\
\text { d. Working with the management } \\
\text { (supervisors, managers, directors) }\end{array}$ & $\begin{array}{l}\mathrm{S} 62 \\
\mathrm{~S} 63\end{array}$ \\
\hline $\begin{array}{l}\text { 7-Work } \\
\text { quantity }\end{array}$ & $\begin{array}{l}\text { a. Amount of work that is done } \\
\text { b. Productive usage of time }\end{array}$ & $\begin{array}{l}S_{71} \\
S_{72}\end{array}$ \\
\hline 8- Security & $\begin{array}{l}\text { a. Other staff/visitors } \\
\text { b. Personal work habits } \\
\text { c. Usage of utilities }\end{array}$ & $\begin{array}{l}\text { S81 } \\
\text { S82 } \\
\text { S83 }\end{array}$ \\
\hline $\begin{array}{l}\text { 9- Personal } \\
\text { learning \& } \\
\text { developmen }\end{array}$ & $\begin{array}{l}\text { a. Occupational knowledge } \\
\text { b. Improving knowledge up to date } \\
\text { nt }\end{array}$ & $\begin{array}{l}\text { S91 } \\
\text { S92 }\end{array}$ \\
\hline 10- Person & $\begin{array}{l}\text { a. Proper dressing } \\
\text { b. Sanitation }\end{array}$ & $\begin{array}{r}\mathrm{S} 101 \\
\mathrm{~S} 102\end{array}$ \\
\hline 11- Leadership & $\begin{array}{l}\text { a. Planning } \\
\text { b. Organizing } \\
\text { c. Cooperative activities } \\
\text { d. Control } \\
\text { e. Ability and tendency to accept } \\
\text { responsibility } \\
\text { f. Accountability } \\
\text { g. Usage of staff through productive } \\
\text { procedures }\end{array}$ & $\begin{array}{l}\text { S111 } \\
\text { S112 } \\
\text { S113 } \\
\text { S114 } \\
\text { S115 }\end{array}$ \\
\hline
\end{tabular}

First step: formation of matrix of criteria and sub-criteria direct impact. Ci represents major criteria of model ( $i$ is the number of criterion). Sij represents sub-criteria

\begin{tabular}{llllllllllll}
\hline \multicolumn{1}{c}{ Table 2: DEMATEL-CRITERIA (DC) direct impact matrix } \\
\hline $\mathrm{DC} \mathrm{C}_{1}$ & $\mathrm{C}_{2}$ & $\mathrm{C}_{3}$ & $\mathrm{C}_{4}$ & $\mathrm{C}_{5}$ & $\mathrm{C}_{6}$ & $\mathrm{C}_{7}$ & $\mathrm{C} 8$ & $\mathrm{C}_{9}$ & $\mathrm{C}_{10}$ & $\mathrm{C}_{11}$ \\
$\mathrm{C}_{1}$ & 0 & 3 & 1 & 4 & 2 & 0 & 1 & 0 & 2 & 3 & 0 \\
$\mathrm{C}_{2}$ & 1 & 0 & 1 & 1 & 1 & 0 & 0 & 4 & 3 & 3 & 1 \\
$\mathrm{C}_{3}$ & 2 & 3 & 0 & 0 & 1 & 1 & 1 & 2 & 4 & 2 & 0
\end{tabular}

\begin{tabular}{llllllllllll}
$\mathrm{C}_{4}$ & 3 & 3 & 4 & 0 & 0 & 0 & 0 & 0 & 0 & 2 & 2 \\
$\mathrm{C}_{5}$ & 3 & 1 & 1 & 1 & 0 & 0 & 3 & 1 & 0 & 1 & 1 \\
$\mathrm{C}_{6}$ & 4 & 3 & 3 & 0 & 2 & 0 & 1 & 3 & 2 & 0 & 3 \\
$\mathrm{C}_{7}$ & 3 & 0 & 2 & 0 & 3 & 0 & 0 & 0 & 0 & 3 & 1 \\
$\mathrm{C}_{8}$ & 4 & 0 & 0 & 0 & 3 & 0 & 1 & 0 & 1 & 1 & 0 \\
$\mathrm{C}_{9}$ & 2 & 0 & 1 & 4 & 3 & 0 & 1 & 1 & 0 & 2 & 1 \\
$\mathrm{C}_{10}$ & 1 & 0 & 0 & 2 & 4 & 3 & 0 & 0 & 3 & 0 & 3 \\
$\mathrm{C}_{11}$ & 1 & 3 & 1 & 2 & 0 & 3 & 4 & 0 & 3 & 3 & 0 \\
\hline
\end{tabular}

$\mathrm{C}_{1}=$ Reliability; $\mathrm{C}_{2}=$ Attitude; $\mathrm{C}_{3}=$ Work quality;

$\mathrm{C}_{4}=$ Innovation; $\mathrm{C}_{5}=$ Judgment; $\mathrm{C}_{6}=$ Cooperation; $\mathrm{C}_{7}=$ Work quantity; $\mathrm{C} 8=$ Security; $\mathrm{C} 9=$ Personal learning \& development; $\mathrm{C}_{10}=$ Person; $\mathrm{C}_{11}=$ Leadership

Second step: All criteria were compared against each other separately and weight of criteria for each paired comparison matrix was calculated according to the geometrical mean method.

$\mathrm{W}_{2}=\left(\mathrm{W}_{21} \ldots \mathrm{W}_{2 \mathrm{n}}\right)$.

Next step: paired comparisons among options considering the criteria.

Assuming that there was no internal correlation between the criteria weight of each option for Index is determined by performing paired comparisons among options considering the criteria (criteria refers to good features of occupational contexts, such as reliability, personal learning, and development etc.). Model options bear great importance regarding each criterion. In order to determine the importance of options regarding a criterion, they were separately compared with each criterion. After formation of paired comparison matrices of options regarding each criterion, the weight of options of each matrix was calculated by the geometrical mean method. For instance, $W_{31}$ shows the weight of options regarding the first criterion.

Criteria and sub-criteria were given weights based on the process in the fourth step and paired comparisons. Since calculations were very complex and timeconsuming, the process was written in MATLAB environment. Calculated criteria and sub-criteria are given in Table 4 .

Third step: it includes assessment of the threshold value and designing the network relationship map.

In designed maps, letters $A$ to $K$ represent the existing members in each matrix.

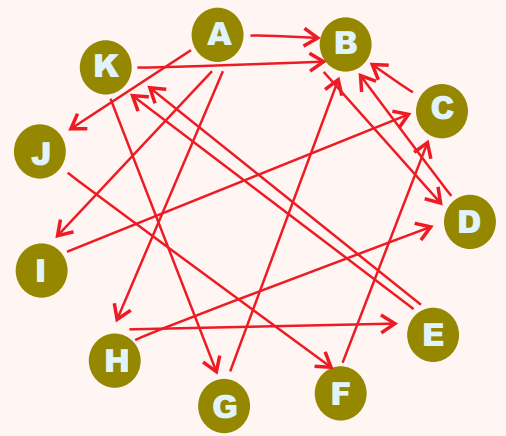

Figure 1: Graph of relationships among criteria 
TECHNOSCIENCE ARTICLE

Table 3: Criteria Weight Matrix for Criteria Paired Comparison Matrix $\mathrm{T}=\mathrm{X}$, Inverse $(\mathrm{I}-\mathrm{X})$

\begin{tabular}{|c|c|c|c|c|c|c|c|c|c|c|c|}
\hline $\mathrm{C}_{1}$ & 0 & $\begin{array}{l}0.0 \\
349 \\
35\end{array}$ & $\begin{array}{l}0.00 \\
67\end{array}$ & $\begin{array}{l}0.04 \\
86\end{array}$ & $\begin{array}{l}0.01 \\
57\end{array}$ & 0 & $\begin{array}{l}0.00 \\
45\end{array}$ & 0 & 0.21 & $\begin{array}{l}0.251 \\
5\end{array}$ & 0 \\
\hline $\mathrm{C}_{2}$ & $\begin{array}{l}0.007 \\
315\end{array}$ & 0 & $\begin{array}{l}0.00 \\
44\end{array}$ & $\begin{array}{l}0.00 \\
44\end{array}$ & $\begin{array}{l}0.00 \\
57\end{array}$ & 0 & $\begin{array}{l}0 \\
5\end{array}$ & $\begin{array}{l}0.047 \\
47\end{array}$ & $\begin{array}{l}0.02 \\
4\end{array}$ & 0 & $\begin{array}{l}0.05 \\
982\end{array}$ \\
\hline$C_{3}$ & $\begin{array}{l}0.020 \\
4\end{array}$ & $\begin{array}{l}0.0 \\
318 \\
15\end{array}$ & 0 & $\begin{array}{l}0.00 \\
00\end{array}$ & $\begin{array}{l}0.00 \\
65\end{array}$ & $\begin{array}{l}0.00 \\
26\end{array}$ & $\begin{array}{l}0.00 \\
47\end{array}$ & $\begin{array}{l}0.017 \\
54\end{array}$ & 0 & $\begin{array}{l}0.027 \\
1\end{array}$ & $\begin{array}{l}0.018 \\
082\end{array}$ \\
\hline $\mathrm{C}_{4}$ & $\begin{array}{l}0.034 \\
545\end{array}$ & $\begin{array}{l}0.0 \\
3534\end{array}$ & $\begin{array}{l}0.04 \\
89\end{array}$ & $\begin{array}{l}0.00 \\
00\end{array}$ & $\begin{array}{l}0.00 \\
00\end{array}$ & 0 & 0 & 0 & $\begin{array}{l}0.06 \\
7\end{array}$ & 0.067 & 0 \\
\hline$C_{5}$ & $\begin{array}{l}0.040 \\
83\end{array}$ & $\begin{array}{l}0.0 \\
066\end{array}$ & $\begin{array}{l}0.00 \\
59\end{array}$ & $\begin{array}{l}0.00 \\
58\end{array}$ & $\begin{array}{l}0.00 \\
00\end{array}$ & 0 & $\begin{array}{l}0.02 \\
93\end{array}$ & $\begin{array}{l}0.010 \\
21\end{array}$ & $\begin{array}{l}0.08 \\
9\end{array}$ & 0 & $\begin{array}{l}0.566 \\
6\end{array}$ \\
\hline C6 & $\begin{array}{l}0.07 \\
18\end{array}$ & $\begin{array}{l}0.0 \\
396\end{array}$ & $\begin{array}{l}0.03 \\
38\end{array}$ & $\begin{array}{l}0.00 \\
00\end{array}$ & $\begin{array}{l}0.02 \\
19\end{array}$ & 0 & $\begin{array}{l}0.00 \\
63\end{array}$ & $\begin{array}{l}0.038 \\
985\end{array}$ & 0 & 0.078 & $\begin{array}{l}0.288 \\
9\end{array}$ \\
\hline$C_{7}$ & $\begin{array}{l}0.034 \\
32\end{array}$ & 0 & $\begin{array}{l}0.01 \\
44\end{array}$ & $\begin{array}{l}0.00 \\
00\end{array}$ & $\begin{array}{l}0.03 \\
02\end{array}$ & 0 & 0 & 0 & $\begin{array}{l}0.00 \\
58\end{array}$ & $\begin{array}{l}0.000 \\
0\end{array}$ & $\begin{array}{l}0.005 \\
8\end{array}$ \\
\hline C8 & $\begin{array}{l}0.055 \\
12\end{array}$ & 0 & 0 & $\begin{array}{l}0.00 \\
00\end{array}$ & $\begin{array}{l}0.03 \\
10\end{array}$ & 0 & $\begin{array}{l}0.00 \\
5\end{array}$ & 0 & $\begin{array}{l}0.00 \\
00\end{array}$ & $\begin{array}{l}0.021 \\
9\end{array}$ & $\begin{array}{l}0.000 \\
0\end{array}$ \\
\hline$\overline{C 9}$ & $\begin{array}{l}0.130 \\
89\end{array}$ & $\begin{array}{l}0.1 \\
221 \\
8\end{array}$ & $\begin{array}{l}0.29 \\
3\end{array}$ & $\begin{array}{l}0.11 \\
94\end{array}$ & $\begin{array}{l}0.01 \\
9429\end{array}$ & 0 & $\begin{array}{l}0.00 \\
66\end{array}$ & $\begin{array}{l}0.005 \\
15\end{array}$ & 0 & $\begin{array}{l}0.035 \\
34\end{array}$ & $\begin{array}{l}0.048 \\
9\end{array}$ \\
\hline $\mathrm{C}_{10}$ & $\begin{array}{l}0.303 \\
03\end{array}$ & $\begin{array}{l}0.2 \\
827 \\
9\end{array}$ & 0 & $\begin{array}{l}0.25 \\
15\end{array}$ & 0 & $\begin{array}{l}0.18 \\
7\end{array}$ & 0 & $\begin{array}{l}0.034 \\
545\end{array}$ & $\begin{array}{l}0.04 \\
083\end{array}$ & 0 & $\begin{array}{l}0.005 \\
9\end{array}$ \\
\hline $\mathrm{C}_{11}$ & $\begin{array}{l}0.303 \\
03\end{array}$ & 0 & $\begin{array}{l}0.28 \\
3\end{array}$ & 0 & $\begin{array}{l}0.05 \\
4982\end{array}$ & $\begin{array}{l}0.13 \\
76\end{array}$ & $\begin{array}{l}0.02 \\
534\end{array}$ & $\begin{array}{l}0.048 \\
9\end{array}$ & $\begin{array}{l}0.07 \\
118\end{array}$ & $\begin{array}{l}0.039 \\
615\end{array}$ & $\begin{array}{l}0 \\
0\end{array}$ \\
\hline
\end{tabular}

Table 4: Criteria and sub-criteria weights

\begin{tabular}{clr}
\hline Criteria & Sub-criteria & Symbol \\
\hline 1-Reliability & a. Punctuality & 0.251 \\
(o.1221) & b. No misuse of sick leaves & 0.145 \\
& c. No misuse of overtime & 0.161 \\
& d. No absence & 0.244 \\
& e. Start working on time & 0.199 \\
2- Attitude & a. Interest in working & 0.226 \\
(o.1132) & b. Commitment to organization & 0.366 \\
& c. Attitude towards other staff & 0.198 \\
& d. Attitude towards other people & 0.210 \\
3-Working & a. Work accomplishment & 0.234 \\
quality & b. Effectiveness & 0.165 \\
(o.o812) & c. Completion of work in allotted time & 0.322 \\
& d. Accuracy and correctness & 0.125 \\
& e. Tidiness & 0.154 \\
4-Innovation & a. Knowing about what has to be done & 0.217 \\
(o.0563) & b. Start duties without special trainings 0.322 \\
& c. Following working duties & 0.461 \\
5-Judgment & a. Use of common reason & 0.468 \\
(o.0254) & b. Proper decision-making at & 0.532 \\
& harsh times & \\
6-Cooperation & a. Working in demanded place and timeo.324 \\
(o.1242) & b. Working in a section or department & 0.222 \\
& c. Working with other sections or & 0.241 \\
& $\quad$ departments & \\
& &
\end{tabular}

Ambient Science, 2016: Vol. 03(Sp1); 10-14 DOI:10.21276/ambi.2016.03.sp1.ta02

d. Working with the management

0.213

(supervisors, managers, directors)

7-Work quantity a. Amount of work that is done $\quad 0.415$

(o.658) b. Productive usage of time $\quad 0.585$

$\begin{array}{ll}\text { 8-Security a. Other staff/visitors } & 0.316\end{array}$

$\begin{array}{lll}(0.0763) & \text { b. Personal work habits } & 0.281\end{array}$

c. Usage of utilities $\quad 0.403$

9-Personal a. Occupational knowledge $\quad 0.648$

$\begin{array}{lll}\text { learning \& } & \text { b. Improving knowledge up to date } \quad 0.352\end{array}$ development

(o.1352)

\begin{tabular}{clc} 
10-Person & a. Proper dressing & 0.632 \\
$($ o.0513) & b. Sanitation & 0.368 \\
11-Leadership & a. Planning & 0.186 \\
$(0.1490)$ & b. Observation & 0.127 \\
& c. Cooperative activities & 0.154 \\
& d. Control & 0.073 \\
& e. Ability and tendency to accept & 0.189 \\
& $\quad$ responsibility & \\
& f. Accountability & 0.227 \\
& g. Usage of staff through productive & 0.047 \\
& procedures & \\
\hline
\end{tabular}

Fourth step: Formation of paired comparison matrix

In the first stage of paired comparison formation, comparisons are made by the obtained graphs from DEMATELsteps.

According to the stages stated in literature (Hossein, 2010; Abbas, 2005) for ANP method and in order to determine internal relationships between criteria and sub-criteria, one has to perform paired comparisons between criteria and sub-criteria according to each criterion and sub-criterion, respectively. DEMATEL technique was employed to avoid the formation of too many paired comparisons. By this technique, paired comparisons were made only between members that affected each other; so that, considering a certain criterion, only those criteria which had an arrow pointed them from the certain criterion were placed in the paired comparison table. If there was only one arrow pointing from one criterion to another, it means that the affecting criterion had a hundred percent impact on the affected criterion (Draker, 1994). The following comparisons were obtained through the design graphs.

\begin{tabular}{lllllllll}
\hline \multicolumn{7}{c}{ Table 5: Criteria paired comparisons (goal based) } \\
\hline $\mathrm{C}_{1}$ & $\mathrm{C}_{1}$ & $\mathrm{C}_{2}$ & $\mathrm{C}_{3}$ & $\mathrm{C}_{4}$ & $\mathrm{C}_{5}$ & $\mathrm{C}_{6}$ & $\mathrm{C}_{7}$ & $\mathrm{C} 8$ \\
$\mathrm{C}_{2}$ & 1 & 2 & $1 / 3$ & $1 / 5$ & $1 / 7$ & $1 / 2$ & $1 / 5$ & $1 / 7$ \\
$\mathrm{C}_{3}$ & $1 / 2$ & 1 & $1 / 2$ & $1 / 2$ & $1 / 5$ & $1 / 5$ & $1 / 7$ & $1 / 8$ \\
$\mathrm{C}_{4}$ & 3 & 2 & 1 & 3 & $1 / 3$ & 5 & $1 / 3$ & 1 \\
$\mathrm{C}_{5}$ & 5 & 2 & $1 / 3$ & 1 & $1 / 8$ & $1 / 3$ & $1 / 5$ & $1 / 5$ \\
$\mathrm{C}_{6}$ & 7 & 5 & 3 & 8 & 1 & 3 & 1 & 1 \\
$\mathrm{C}_{7}$ & 2 & 5 & $1 / 5$ & 3 & $1 / 3$ & 1 & $1 / 3$ & $1 / 3$ \\
$\mathrm{C}_{8}$ & 5 & 7 & 3 & 5 & 1 & 3 & 1 & $1 / 3$ \\
$\mathrm{C}_{9}$ & 7 & 8 & 1 & 5 & 1 & 3 & 3 & 1 \\
\hline
\end{tabular}

$\mathrm{C}_{1}=$ method covering procedure; $\mathrm{C}_{2}=$ measurability; $\mathrm{C}_{3}=$ Involvement level; $C_{4}=$ required facilities for each method; $C_{5}=$ 


\begin{tabular}{|c|c|c|c|c|c|c|c|c|c|c|c|c|c|c|c|c|c|c|c|}
\hline Factor & $\mathrm{C}_{1}$ & $\mathrm{C}_{2}$ & $C_{3}$ & $\mathrm{C}_{4}$ & $\mathrm{C}_{5}$ & C6 & $\mathrm{C}_{7}$ & C8 & $\mathrm{C}_{9}$ & $\mathrm{C}_{10}$ & $\mathrm{C}_{11}$ & $\mathrm{D}_{1}$ & $\mathrm{D}_{2}$ & $\mathrm{D}_{3}$ & $\mathrm{D}_{4}$ & $\mathrm{D}_{5}$ & D6 & $\mathrm{D}_{7}$ & D8 \\
\hline$\overline{C_{1}}$ & 0.040 & 0.119 & 0.047 & 0.007 & 0.123 & 0.036 & 0.003 & 0.079 & 0.122 & 0.002 & 0.002 & 0.003 & 0.018 & 0.097 & 0.027 & 0.007 & 0.014 & 0.011 & 0.106 \\
\hline $\mathrm{C}_{2}$ & 0.094 & 0.025 & 0.048 & 0.005 & 0.091 & 0.023 & 0.001 & 0.015 & 0.023 & 0.002 & 0.001 & 0.002 & 0.014 & 0.015 & 0.009 & 0.005 & 0.004 & 0.002 & 0.095 \\
\hline$C_{3}$ & 0.078 & 0.113 & 0.025 & 0.001 & 0.018 & 0.063 & 0.004 & 0.007 & 0.011 & 0.000 & 0.001 & 0.002 & 0.003 & 0.092 & 0.090 & 0.006 & 0.022 & 0.018 & 0.017 \\
\hline $\mathrm{C}_{4}$ & 0.017 & 0.023 & 0.099 & 0.011 & 0.085 & 0.109 & 0.006 & 0.012 & 0.013 & 0.041 & 0.007 & 0.008 & 0.019 & 0.022 & 0.040 & 0.034 & 0.015 & 0.007 & 0.063 \\
\hline$C_{5}$ & 0.066 & 0.071 & 0.037 & 0.051 & 0.031 & 0.117 & 0.006 & 0.093 & 0.090 & 0.013 & 0.009 & 0.018 & 0.115 & 0.033 & 0.031 & 0.038 & 0.016 & 0.007 & 0.101 \\
\hline C6 & 0.018 & 0.019 & 0.098 & 0.008 & 0.011 & 0.010 & 0.017 & 0.010 & 0.004 & 0.002 & 0.006 & 0.010 & 0.009 & 0.033 & 0.026 & 0.079 & 0.085 & 0.013 & 0.011 \\
\hline$C_{7}$ & 0.015 & 0.020 & 0.105 & 0.001 & 0.010 & 0.013 & 0.019 & 0.081 & 0.009 & 0.001 & 0.001 & 0.005 & 0.002 & 0.018 & 0.125 & 0.007 & 0.120 & 0.100 & 0.006 \\
\hline C8 & 0.087 & 0.106 & 0.084 & 0.006 & 0.101 & 0.081 & 0.003 & 0.021 & 0.096 & 0.001 & 0.001 & 0.003 & 0.013 & 0.027 & 0.059 & 0.009 & 0.015 & 0.009 & 0.029 \\
\hline $\mathrm{C}_{9}$ & 0.120 & 0.100 & 0.093 & 0.005 & 0.087 & 0.086 & 0.005 & 0.094 & 0.031 & 0.002 & 0.001 & 0.003 & 0.014 & 0.105 & 0.103 & 0.010 & 0.026 & 0.020 & 0.084 \\
\hline Cio & 0.028 & 0.029 & 0.087 & 0.107 & 0.022 & 0.035 & 0.018 & 0.014 & 0.010 & 0.027 & 0.116 & 0.097 & 0.127 & 0.130 & 0.132 & 0.097 & 0.029 & 0.026 & 0.059 \\
\hline C11 & 0.015 & 0.016 & 0.065 & 0.089 & 0.011 & 0.022 & 0.005 & 0.004 & 0.003 & 0.059 & o.o11 & 0.013 & 0.087 & 0.102 & 0.087 & 0.014 & 0.020 & 0.018 & 0.011 \\
\hline D1 & 0.025 & 0.024 & 0.082 & 0.066 & 0.016 & 0.024 & 0.051 & 0.014 & 0.006 & 0.093 & 0.095 & 0.022 & 0.103 & 0.123 & 0.129 & 0.075 & 0.031 & 0.028 & 0.018 \\
\hline D1 & 0.022 & 0.021 & 0.049 & 0.081 & 0.017 & 0.112 & 0.016 & 0.011 & 0.006 & 0.092 & 0.060 & 0.093 & 0.029 & 0.117 & 0.103 & 0.082 & 0.031 & 0.022 & 0.019 \\
\hline $\mathrm{D}_{2}$ & 0.090 & 0.075 & 0.062 & 0.001 & 0.016 & 0.008 & 0.016 & 0.009 & 0.012 & 0.001 & 0.001 & 0.004 & 0.003 & 0.019 & 0.097 & 0.001 & 0.097 & 0.096 & 0.016 \\
\hline$D_{3}$ & 0.012 & 0.015 & 0.093 & 0.000 & 0.003 & 0.006 & 0.019 & 0.003 & 0.002 & 0.000 & 0.000 & 0.005 & 0.001 & 0.068 & 0.028 & 0.001 & 0.115 & 0.114 & 0.004 \\
\hline$D_{4}$ & 0.130 & 0.113 & 0.141 & 0.100 & 0.118 & 0.056 & 0.113 & 0.116 & 0.038 & 0.023 & 0.072 & 0.091 & 0.110 & 0.134 & 0.143 & 0.022 & 0.042 & 0.036 & 0.114 \\
\hline D5 & 0.009 & 0.012 & 0.098 & 0.003 & 0.003 & 0.008 & 0.098 & 0.009 & 0.002 & 0.004 & 0.004 & 0.040 & 0.005 & 0.018 & 0.087 & 0.004 & 0.027 & 0.096 & 0.004 \\
\hline D6 & 0.003 & 0.004 & 0.021 & 0.000 & 0.001 & 0.004 & 0.087 & 0.007 & 0.002 & 0.000 & 0.000 & 0.004 & 0.001 & 0.007 & 0.075 & 0.001 & 0.095 & 0.022 & 0.016 \\
\hline D7 & 0.017 & 0.069 & 0.022 & 0.005 & 0.014 & 0.110 & 0.003 & 0.010 & 0.081 & 0.005 & 0.004 & 0.006 & 0.052 & 0.018 & 0.016 & 0.012 & 0.012 & 0.004 & 0.014 \\
\hline D8 & 0.012 & 0.015 & 0.093 & 0.000 & 0.003 & 0.006 & 0.019 & 0.003 & 0.002 & 0.000 & 0.000 & 0.005 & 0.001 & 0.068 & 0.028 & 0.001 & 0.115 & 0.114 & 0.004 \\
\hline
\end{tabular}

Figure 2: Harmonic super matrix 1

strategic consistency of method with organization goals; $\mathrm{C} 6=$ nature of services; $C_{7}=$ consistency of method with internal and external environments of organization; $\mathrm{C} 8=$ marketing researches results.

4.3.4. Fifth step: formation of super matrix

A super matrix was formed using the discussed paired comparisons (Fig. 2).

\section{Discussion:}

In the present study to determine the internal communication standards, we used DEMATEL techniques.Further, using the comparison table test, interconnections criteria were found.DEMATEL and AHP combination of techniques were used to reduce the number of tables paired comparisons in determining internal communications and increase the efficiency of the AHP technique.The results show that the criteria of leadership, partnership and reliability have high-impact on performance and three other criteria i.e., the person, work quality and attitude were less than expected.

Finally, the results of the prioritization criteria were investigated and evaluated. An analysis of the results was performed using charts. As per the results, the index of the first rank leadership and attitude indicator was ranked last. The results of the ranking indicators and subindicators specif ied curve.

Acknowledgements:

We thank the staffs of Iran Air Homa for their cooperation during the study.

\section{References:}

Abbas, F. (2001): Educational assessment, Tehran: The Organization for Researching and Composing University textbooks in the Humanities.

Abbas, A. (2005): Advanced Human Resources Management, second edition, Tehran: Samt Publications

Amir, F. (2006): Organizational performance assessment, Tadbir Journal, No. 26
Assadollah, F. (2008): Role of staff performance assessment on optimization of human resources. MSc thesis. Business Management, University of Tehran.

Draker, P. (1994): Future Management. Translated by Abdoreza Rezaei Nejad, Resa Cultural Services Institute.

Jafar, G. (2008): Assessment of job enrichment impact on staff performance improvement. MSc thesis, Businesss Management, Mirzaei, University of Tehran.

Hosein, A.S. (1999): Training and optimization of human resources, Tehran. Iranian Organization for Development and Renovation of Industries Publications

Hossein, Z. (2010): Performance assessment effects on optimization of human resources in Iranian Police. MSc thesis, University of Shiraz.

Janie, R.K. \& Triandis, H.C. (2002): Managing the unmanageable. translated and published by Defence Industries Research and Education Institute, second edition, Tehran.

Jones, J.Y. \& Burly, W. (2000): 360- degree feedback of strategies, methods and procedures for managers. translated by EsmailAsgharpoor and GholamrezaTaleghani, Tehran: Sapco Publications

Mansour, A. (2008): Role of staff performance assessment on improvement of human resources. MSc thesis. Business Management, TarbiatModarres University.

Mark, M. (1999): Human Resources Management. translated by Farideh Alaqa, Tehran: Governmental management training centre publications.

Michael, A. (2002): Strategic management of human resources. translated by Mohammad Arabi and Davood Izadi, Cultural Researches Office, Tehran.

Naseer, P.S. (2000): Assessment attitudes on education effectiveness, Tadbir J., No. 160.

Nejad, M. (2009): Performance assessment on leadership management. MSc thesis, University of Tehran

Reza, J. (2003): Morale and satisfaction of work, Tehran. Governmental management training centre publications

Saaty, T.L. (1980) The Analytic Hierarchy Process, New York: McGraw Hill. International. Translated to Russian, Portuguese, and Chinese, Revised editions, Paperback (1996, 2000), Pittsburgh: RWS Publications. 